\title{
Analytic Wave Front Sets of the Riemann Functions of Hyperbolic Mixed Problems in a Quarter-Space
}

By

\author{
Seiichiro WAKABAYASHI*
}

\section{Introduction}

Duff [3] studied the location and structures of singularities of reflected Riemann functions for hyperbolic mixed problems with constant coefficients in a quarter-space making use of the stationary phase method. However, it seems that it is difficult to apply the method to the study of Riemann functions of more general hyperbolic mixed problems. Matsumura [6] gave an inner estimate of the location of singularities of reflected Riemann functions which correspond to reflected waves, making use of the localization method developed by Atiyah, Bott and Garding [2] and Hörmander [4]. In [9] the author proved a localization theorem describing the location of singularities of reflected Riemann functions which correspond to reflected waves, lateral waves and boundary waves. Tsuji [8] also studied the same problem in the cases where operators are homogeneous and obtained similar results. On the other hand outer estimates of singular supports of fundamental solutions for the Cauchy problems were also given in [2]. In this paper we shall be concerned with outer estimates of singular supports of reflected Riemann functions for hyperbolic mixed problems.

Now let us state our problems and assumptions. Let $\mathbf{R}^{n}$ denote the $n$-dimensional Euclidean space and $\Xi^{n}$ its real dual space and write $x^{\prime}=\left(x_{1}, \ldots, x_{n-1}\right), x^{\prime \prime}=\left(x_{2}, \ldots, x_{n}\right)$ for the coordinate $x=\left(x_{1}, \ldots, x_{n}\right)$ in $\mathbb{R}^{n}$ and $\xi^{\prime}=\left(\xi_{1}, \ldots, \xi_{n-1}\right), \xi^{\prime \prime}=\left(\xi_{2}, \ldots, \xi_{n}\right), \tilde{\xi}=\left(\xi, \xi_{n+1}\right)$ for the dual coordinate

Communicated by S. Matsuura, June 17, 1975.

* Department of Mathematics, Tokyo University of Education, Tokyo, Japan. 
$\xi=\left(\xi_{1}, \ldots, \xi_{n}\right)$. We shall also denote by $\mathbb{R}_{+}^{n}$ the half-space $\left\{x=\left(x^{\prime}, x_{n}\right)\right.$ $\left.\in \mathbb{R}^{n} ; x_{n}>0\right\}$. For differentiation we will use the symbol $D=i^{-1}(\partial /$ $\left.\partial x_{1}, \ldots, \partial / \partial x_{n}\right)$. Let $P=P(\xi)$ be a hyperbolic polynomial of order $m$ of $n$ variables $\xi$ with respect to $\vartheta=(1,0, \ldots, 0)$ in $\Xi^{n}$ in the sense of Gårding, i.e. $P^{0}(\vartheta) \neq 0$ and $P(\xi+s \vartheta) \neq 0$ when $\xi$ is real and $\operatorname{Im} s<-\gamma_{0}$, where $P^{0}$ denotes the principal part of $P$. Moreover we assume that $P^{0}(0,1) \neq 0$. We consider the mixed initial-boundary value problem for the hyperbolic operator $P(D)$ in a quarter-space

$$
\begin{array}{ll}
P(D) u(x)=f(x), & x \in \mathbb{R}_{+}^{n}, \quad x_{1}>0, \\
\left(D_{1}^{k} u\right)\left(0, x^{\prime \prime}\right)=0, & 0 \leq k \leq m-1, \quad x_{n}>0, \\
\left.B_{j}(D) u(x)\right|_{x_{n}=0}=0, & 1 \leq j \leq l, \quad x_{1}>0 .
\end{array}
$$

Here the $B_{j}(D)$ are boundary operators with constant coefficients and the number $l$ of boundary conditions is equal to that of the roots with positive imaginary part of the equation $P\left(\xi^{\prime}-i \gamma \vartheta^{\prime}, \lambda\right)=0$ with respect to $\lambda$, where $\gamma>\gamma_{0}$.

Let us denote by $\Gamma=\Gamma(P, \vartheta)\left(\subset \Xi^{n}\right)$ the component of the set $\left\{\xi \in \Xi^{n} ; P^{0}(\xi) \neq 0\right\}$ which contains $\vartheta$ and put $\Gamma_{0}=\left\{\xi^{\prime} \in \Xi^{n-1} ;\left(\xi^{\prime}, 0\right) \in \Gamma\right\}$. When $\xi^{\prime} \in \Xi^{n-1}-i \gamma_{0} \vartheta^{\prime}-i \Gamma_{0}$, we can denote the roots of $P\left(\xi^{\prime}, \lambda\right)=0$ with respect to $\lambda$ by $\lambda_{1}^{+}\left(\xi^{\prime}\right), \ldots, \lambda_{l}^{+}\left(\xi^{\prime}\right), \lambda_{1}^{-}\left(\xi^{\prime}\right), \ldots, \lambda_{m-l}^{-}\left(\xi^{\prime}\right)$, which are enumerated so that

$$
\begin{array}{ll}
\operatorname{Im} \lambda_{k}^{+}\left(\xi^{\prime}\right)>0, & 1 \leqq k \leqq l, \\
\operatorname{Im} \lambda_{k}^{-}\left(\xi^{\prime}\right)<0, & 1 \leqq k \leqq m-l .
\end{array}
$$

Put

$$
P_{+}\left(\xi^{\prime}, \lambda\right)=\prod_{j=1}^{l}\left(\lambda-\lambda_{j}^{+}\left(\xi^{\prime}\right)\right), \quad \xi^{\prime} \in \Xi^{n-1}-i \gamma_{0} \vartheta^{\prime}-i \Gamma_{0}
$$

We now define the Lopatinski determinant for the system $\left\{P, B_{j}\right\}$ by

$$
\begin{array}{r}
R\left(\xi^{\prime}\right)=\operatorname{det}\left(\frac{1}{2 \pi i} \oint \frac{B_{j}\left(\xi^{\prime}, \lambda\right) \lambda^{k-1}}{P_{+}\left(\xi^{\prime}, \lambda\right)} d \lambda\right)_{j, k=1, \ldots, l}, \\
\xi^{\prime} \in \Xi^{n-1}-i \gamma_{0} \vartheta^{\prime}-i \Gamma_{0} .
\end{array}
$$

We state the assumptions that we impose on $\left\{P, B_{j}\right\}$ : 


$$
P(\xi)=p_{1}(\xi)^{v_{1} \cdots} p_{q}(\xi)^{v_{q}},
$$

where the $p_{j}(\xi)$ are distinct strictly hyperbolic polynomials with respect to $\vartheta$.

(A.2) The system $\left\{P, B_{j}\right\}$ is $\mathscr{E}$-well posed, i.c.

$$
\begin{aligned}
& R\left(\xi^{\prime}+s \vartheta^{\prime}\right) \neq 0 \text { for } \xi^{\prime} \in \Xi^{n-1} \text { and } \operatorname{Im} s<-\gamma_{1}, \\
& \widetilde{R}_{0}\left(\vartheta^{\prime}\right) \neq 0
\end{aligned}
$$

where $\widetilde{R}_{0}\left(\xi^{\prime}\right)$ denotes the principal part of $R\left(\xi^{\prime}\right)$ defined by (2.2) (see Sakamoto [7]).

Now we can construct the Riemann function $G(x, y)$ for $\left\{P, B_{j}\right\}$ which describes the propagation of waves produced by unit impulse given at position $y=\left(0, y_{2}, \ldots, y_{n}\right)$ in $\mathbb{R}_{+}^{n}$ (see [7], [8]). Write

$$
\begin{aligned}
G(x, y)=E(x-y)-F(x, y), \quad x & \in \mathbb{R}_{+}^{n}, \quad x_{1}>0, \\
y & =\left(0, y_{2}, \ldots, y_{n}\right) \in \mathbb{R}_{+}^{n},
\end{aligned}
$$

where $E(x)$ is the fundamental solution represented by

$$
E(x)=(2 \pi)^{-n} \int_{\Xi^{n-i \eta}} \exp [i x \cdot \xi] P(\xi)^{-1} d \xi, \quad \eta \in \gamma_{0} \vartheta+\Gamma .
$$

Then the reflected Riemann function $F(x, y)$ is written in the form

$$
\begin{aligned}
& F(x, y)=(2 \pi)^{-(n+1)} \int_{\Sigma^{n+1}-i \eta} \frac{1}{i} \sum_{j, k=1}^{l} \exp \left[i \left\{\left(x^{\prime}-y^{\prime}\right) \cdot \xi^{\prime}\right.\right. \\
& \left.\left.-y_{n} \xi_{n}+x_{n} \xi_{n+1}\right\}\right] \frac{R_{j k}\left(\xi^{\prime}\right) B_{k}(\xi) \xi_{n+1}^{j-1}}{R\left(\xi^{\prime}\right) P_{+}\left(\xi^{\prime}, \xi_{n+1}\right) P(\xi)} d \tilde{\xi}, \\
& \\
& \quad \eta \in \gamma_{1} \vartheta+\Gamma, \eta^{\prime} \in \gamma_{1} \vartheta^{\prime}+\Gamma_{0}, \eta_{n+1}=0,
\end{aligned}
$$

where

$$
\text { (1.11) } R_{j k}\left(\xi^{\prime}\right)=(k, j) \text { - cofactor of }\left(\frac{1}{2 \pi i} \oint \frac{B_{j}\left(\xi^{\prime}, \lambda\right) \lambda^{k-1}}{P_{+}\left(\xi^{\prime}, \lambda\right)} d \lambda\right)_{j, k=1, \ldots, l} \text {. }
$$

$F(x, y)$ has to be interpreted in the sense of distribution with respect to $(x, y)$ in $\mathbb{R}_{+}^{n} \times \mathbb{R}_{+}^{n}$. We put

$$
\widetilde{\Gamma}\left(x^{\prime}, y_{n}, x_{n}\right)=F\left(x, 0, y_{n}\right)
$$


and regard $\tilde{F}\left(x^{\prime}, y_{n}, x_{n}\right)$ as a distribution on $X=\mathbf{R}^{n-1} \times \mathbb{R}_{+}^{1} \times \mathbb{R}_{+}^{1}$.

In [2] Atiyah, Bott and Gårding proved the following

Proposition 1.1. If $P(\xi)$ is a hyperbolic polynomial with respect to $\vartheta$, then

$$
\cup_{\xi \in \bar{\Xi}^{n}\{\{0\}} \operatorname{supp} E_{\xi} \times\{\xi\} \subset W F(E) \subset W F_{A}(E) \subset \cup_{\xi \in \bar{z}^{n} \backslash\{0\}} K_{\xi} \times\{\xi\}
$$

holds and, moreover,

$$
\overline{c h}\left[\operatorname{supp} E_{\xi 0}\right]=K_{\xi 0}
$$

holds. Here

$$
\begin{gathered}
E_{\xi^{0}}(x)=(2 \pi)^{-n} \int_{\Sigma^{n-i \eta}} \exp [i x \cdot \xi] P_{\xi^{0}}(\xi)^{-1} d \xi, \quad \eta \in \gamma_{0} \vartheta+\Gamma \\
K_{\xi^{0}}=\left\{x \in \mathbf{R}^{n} ; x \cdot \eta \geqq 0 \text { for all } \eta \in \Gamma\left(P_{\xi^{0}}, \vartheta\right)\right\}
\end{gathered}
$$

where the localization $P_{\xi^{0}}$ of $P$ at $\xi^{0}$ is defined by

$$
v^{m} P\left(v^{-1} \xi^{0}+\eta\right)=v^{p} P_{\xi^{0}}(\eta)+O\left(v^{p+1}\right) \quad \text { as } \quad v \rightarrow 0 .
$$

Our aim is to obtain results corresponding to Proposition 1.1 for $\tilde{F}\left(x^{\prime}, y_{n}, x_{n}\right)$ under the conditions (A.1) and (A.2).

The remainder of this paper is organized as follows. In $\S 2$ we shall state results obtained in [9] and give some remarks. We shall investigate some properties of the Lopatinski determinant $R\left(\xi^{\prime}\right)$ in $\S 3$. An outer estimate of $W F_{A}(\widetilde{F})$ will be given in $\S 4$. In $\S 5$ we shall give some remarks and examples.

The author would like to express his sincere gratitude to Professor M. Matsumura for many valuable suggestions.

\section{Localization Theorem}

Put

$$
\dot{\Gamma}=\left\{\xi^{\prime} \in \Xi^{n-1} ;\left(\xi^{\prime}, \xi_{n}\right) \in \Gamma \text { for some } \xi_{n} \in \Xi\right\}
$$

$\dot{\Gamma}$ coincides with one defined in [7], [9]. 
Lemma $2.1([7]) . \quad R\left(\xi^{\prime}\right)$ is holomorphic in $\Xi^{n-1}-i \gamma_{0} \vartheta^{\prime}-i \Gamma$.

Lemma 2.2 ([7]). Let $K$ be a compact set in $\Xi^{n-1}-i \dot{\Gamma}$, then there exists $T_{K}>0$ such that

$$
R\left(t \xi^{\prime}\right)=t^{h_{0}}\left\{\widetilde{R}_{0}\left(\xi^{\prime}\right)+t^{-1} \widetilde{R}_{1}\left(\xi^{\prime}\right)+t^{-2} \widetilde{R}_{2}\left(\xi^{\prime}\right)+\cdots\right\}
$$

whose convergence is uniform in $K \times\left\{t>T_{K}\right\}$, where

(i) $\left\{\tilde{R}_{j}\left(\xi^{\prime}\right)\right\}$ are holomorphic in $\dot{\Gamma}=\cup_{z \in \mathbf{C} \backslash\{0\}} z\left(\Xi^{n-1}-i \dot{\Gamma}\right)$,

(ii) $\widetilde{R}_{j}\left(t \xi^{\prime}\right)=t^{h_{0}-j} \tilde{R}_{j}\left(\xi^{\prime}\right) \quad$ for $\xi^{\prime} \in \dot{\Gamma}, \quad t \in \mathbf{C} \backslash\{0\}$,

(iii) $\widetilde{R}_{0}\left(\xi^{\prime}\right) \not \equiv 0$ and $h_{0}$ is an integer.

Let us denote by $\dot{\Sigma}\left(\subset \Xi^{n-1}\right)$ the component of the set $\left\{\xi^{\prime} \in \dot{\Gamma}\right.$; $\left.\tilde{R}_{0}\left(-i \xi^{\prime}\right) \neq 0\right\}$ which contains $\vartheta^{\prime}$.

Lemma 2.3. $\dot{\Sigma}$ is an open convex cone and

$$
R\left(\xi^{\prime}\right) \neq 0 \quad \text { for } \quad \xi^{\prime} \in \Xi^{n-1}-i \gamma_{1} \vartheta^{\prime}-i \dot{\Sigma} .
$$

Remark. $\dot{\Sigma}$ coincides with one defined in [7], [9].

Proof. We first prove that $\dot{\Sigma}$ is star-shaped with respect to $\vartheta^{\prime}$. Put

$$
f\left(t, \eta^{\prime}\right)=\widetilde{R}_{0}\left(t \eta^{\prime}-i \vartheta^{\prime}\right), \quad \operatorname{Im} t \leqq 0, \quad \eta^{\prime} \in \dot{\Sigma} .
$$

Then $f\left(t, \eta^{\prime}\right)$ is holomorphic for $\operatorname{Im} t<0$ and continuous for $\operatorname{Im} t \leqq 0$, $\eta^{\prime} \in \dot{\Sigma}$. Since $\dot{\Sigma}$ is connected, there exists a continuous function $\eta^{\prime}(\theta)$, $0 \leqq \theta \leqq 1$, such that $\eta^{\prime}(\theta) \in \dot{\Sigma}, \eta^{\prime}(0)=\eta^{\prime}$ and $\eta^{\prime}(1)=\vartheta^{\prime}$. From Lemma 2.1 in [7] it follows that

$$
f\left(t, \eta^{\prime}(\theta)\right) \neq 0 \quad \text { when } \operatorname{Im} t=0 \text { and } 0 \leqq \theta \leqq 1 .
$$

Since the set $\left\{\eta^{\prime}(\theta) ; 0 \leqq \theta \leqq 1\right\}$ is compact in $\dot{\Sigma}$, we see that

$$
f\left(t, \eta^{\prime}(\theta)\right) \neq 0 \quad \text { when } \quad|t| \geqq T, \quad \operatorname{Im} t \leqq 0 \quad \text { and } \quad 0 \leqq \theta \leqq 1,
$$

where $T=\max _{0 \leqq \theta \leqq 1}\left\{2 / \operatorname{dis}\left(-i \eta^{\prime}(\theta),\left\{\zeta^{\prime} \in \Xi^{n-1}-i \dot{\Gamma} ; \widetilde{R}_{0}\left(\zeta^{\prime}\right)=0\right\} U-i \partial \dot{\Gamma}\right)\right\}$. This follows from

$$
\widetilde{R}_{0}\left(t \eta^{\prime}(\theta)-i \vartheta^{\prime}\right)=(i t)^{h_{0}} \widetilde{R}_{0}\left(-i \eta^{\prime}(\theta)-t^{-1} \vartheta^{\prime}\right)
$$


Let us assume that there exists a complex number $t_{0}$ such that $\operatorname{Im} t_{0} \leqq 0$ and $f\left(t_{0}, \eta^{\prime}\right)=0$. Then (2.4) and (2.5) imply that $\operatorname{Im} t_{0}<0$ and $0<\left|t_{0}\right|$ $<T$. Moreover from (2.4) and (2.5) there exists a continuous function $t(\theta)$ such that $f\left(t(\theta), \eta^{\prime}(\theta)\right)=0, \operatorname{Im} t(\theta)<0,|t(\theta)|<T$ and $t(0)=t_{0}$. This is contrary to $f\left(t, \vartheta^{\prime}\right) \neq 0$ for $\operatorname{Im} t \leqq 0$. Therefore we have

$$
f\left(t, \eta^{\prime}\right)=\widetilde{R}_{0}\left(t \eta^{\prime}-i \vartheta^{\prime}\right) \neq 0 \quad \text { for } \quad \operatorname{Im} t \leqq 0 .
$$

It easily follows from (2.6) that $\Sigma$ is star-shaped with respect to $\vartheta^{\prime}$ and that $\dot{\Sigma}$ coincides with one defined in [7]. Thus (2.3) follows from Lemma 2.2 in [7]. Putting $f\left(t, \eta^{\prime}\right)=\widetilde{R}_{0}\left(t \eta^{\prime}-i \eta^{0 \prime}\right)$, where $\eta^{\prime}, \eta^{0 \prime} \in \dot{\Sigma}$ and $\operatorname{Im} t \leqq 0$, and applying the same argument to $f\left(t, \eta^{\prime}\right)$ we can prove the convexity of $\dot{\Sigma}$.

Q.E.D.

Let $\xi^{0}$ be arbitrarily fixed in $\Xi^{n-1} \backslash\{0\}$ and let $\left\{j_{k}\right\}_{1 \leqq k \leqq r_{1}}$ be the set of suffixes so that $p_{j_{k}}^{0}\left(\xi^{0^{\prime}}, \mu\right)=0$ has a real multiple root $\mu_{k}$. We define $\dot{\Gamma}_{\xi^{0}}$, by

$$
\dot{\Gamma}_{\xi^{0},} \times \Xi=\cap_{k=1}^{r_{1}} \Gamma\left(p_{j_{h}\left(\xi^{0}, \mu_{k}\right)}, \vartheta\right) .^{\dagger}
$$

Lemma 2.4 (Lemma 2.6 in [9]). For any compact set $K$ in $\Xi^{n-1}$ $-i \gamma_{0} \vartheta^{\prime}-i \dot{\Gamma}$ and any non-negative integer $N$ there exists $\varepsilon>0$ such that if $\eta^{\prime} \in K$ and $0<v<\varepsilon$,

$$
v^{h_{1}} R\left(v^{-1} \xi^{0^{\prime}}+\eta^{\prime}\right)=\sum_{j=0}^{N} Q_{j}\left(\eta^{\prime}\right) v^{j / L}+O\left(v^{(N+1) / L}\right),
$$

where $Q_{0}\left(\eta^{\prime}\right) \not \equiv 0, L$ is a positive integer and $h_{1}$ is a rational number. Moreover the $Q_{j}\left(\eta^{\prime}\right)$ are holomorphic in $\Xi^{n-1}-i \gamma_{0} \vartheta^{\prime}-i \dot{\Gamma}_{\xi^{0}}$.

Let $Q_{0}^{0}\left(\eta^{\prime}\right)$ be the principal part of $Q_{0}\left(\eta^{\prime}\right)$ (see [9]). We denote by $\dot{\Sigma}_{\xi^{0}}$ the component of the set $\left\{\eta^{\prime} \in \dot{\Gamma}_{\xi^{\prime}} ; Q_{0}^{0}\left(-i \eta^{\prime}\right) \neq 0\right\}$ which contains $\vartheta^{\prime} . \quad \dot{\Sigma}_{\xi^{0}}$, coincides with one defined in [9]. Then by the same argument as in Lemma 2.3 we have the following

Lemma 2.5. $\dot{\Sigma}_{\xi^{0}}$, is an open convex cone and

$$
Q_{0}\left(\eta^{\prime}\right) \neq 0 \quad \text { for } \quad \eta^{\prime} \in \Xi^{n-1}-i \gamma_{1} \vartheta^{\prime}-i \dot{\Sigma}_{\xi 0^{\prime}}
$$

$\dagger$ If $r_{1}=0$, then we put $\dot{L}_{\natural^{\prime \prime}}=\Xi^{n-1}$. 


$$
Q_{0}^{0}\left(\eta^{\prime}\right) \neq 0 \quad \text { for } \quad \eta^{\prime} \in \Xi^{n-1}-i \dot{\Sigma}_{\xi^{\circ}}
$$

Let $\xi_{n+1}^{0} \in \Xi$ and let $\left\{s_{k}\right\}_{1 \leqq k \leqq r_{0}}$ be the set of suffixes so that $p_{s_{k}}^{0}\left(\xi^{0 \prime}\right.$, $\left.\xi_{n+1}^{0}\right)=0$ and $\left.\frac{\partial p_{s_{k}}^{0}}{\partial \xi_{1}}\left(\xi^{0^{\prime}}, \xi_{n+1}^{0}\right) \cdot \frac{\partial p_{s_{k}}^{0}}{\partial \mu}\left(\xi^{0 \prime}, \mu\right)\right|_{\mu=\xi_{n+1}^{0}}>0$. This implies that $\xi_{n+1}^{0}$ is a real simple root of $p_{s_{k}}^{0}\left(\xi^{0^{\prime}}, \mu\right)=0$ which corresponds to a root with positive imaginary part of $p_{s_{k}}^{0}\left(\xi^{0 \prime}-i \gamma \vartheta^{\prime}, \mu\right)=0, \gamma>0$. Define

$$
\tilde{\Gamma}_{\left(\xi^{0^{0}}, \xi_{n+1}^{0}\right)}=\cap_{k=1}^{r_{0}}\left\{\left\{\tilde{\xi} \in \Xi^{n+1} ;\left(\xi^{\prime}, \xi_{n+1}\right) \in \Gamma\left(p_{s_{k}\left(\xi^{0}, \xi_{n+1}^{0}\right)}, \vartheta\right)\right\}\right.
$$

Let $\tilde{\xi}^{0}$ be arbitrarily fixed in $\Xi^{n+1} \backslash\{0\}$ and put

$$
\Gamma_{\xi^{0}}=\left(\Gamma\left(P_{\xi^{0},}, \vartheta\right) \times \Xi\right) \cap \widetilde{\Gamma}_{\left(\xi^{0}, \xi_{n+1}^{0}\right)} \cap\left(\dot{\Sigma}_{\xi^{0}} \times \Xi^{2}\right) .
$$

Here we put, if $\xi^{0 \prime}=0$,

$$
\dot{\Sigma}_{\xi^{\prime}}=\dot{\Sigma}\left(\dot{\Gamma}_{\xi^{0}}=\dot{\Gamma}\right)
$$

$$
\tilde{\Gamma}_{\left(\xi^{\left.0^{\prime}, \xi_{n+1}^{0}\right)}\right.}=\left\{\begin{array}{l}
\Xi^{n+1} \quad \text { if } \quad \xi_{n+1}^{0} \neq 0, \\
\left\{\tilde{\xi} \in \Xi^{n+1} ;\left(\xi^{\prime}, \xi_{n+1}\right) \in \Gamma(P, \vartheta)\right\} \quad \text { otherwise. }
\end{array}\right.
$$

In [9] the author proved the following

Theorem 2.6. Assume that the conditions (A.1) and (A.2) are satisfied and that $\xi^{0} \in \Xi^{n+1}$.

Then we have

$$
\begin{gathered}
t^{p_{0}} \exp \left[-i t\left(x^{\prime} \cdot \xi^{0 \prime}-y_{n} \xi_{n}^{0}+x_{n} \xi_{n+1}^{0}\right)\right] \widetilde{F}\left(x^{\prime}, y_{n}, x_{n}\right) \\
\sim \sum_{j=0}^{\infty} \widetilde{F}_{\tilde{\xi}^{0}, j}\left(x^{\prime}, y_{n}, x_{n}\right) t^{-j / L},
\end{gathered}
$$

where $p_{0}$ is a rational number and $L$ is a positive integer. Here (2.15) implies that

$$
\begin{gathered}
t^{N / L}\left\{t^{p_{0}} \exp \left[-i t\left(x^{\prime} \cdot \xi^{0^{\prime}}-y_{n} \xi_{n}^{0}+x_{n} \xi_{n+1}^{0}\right)\right] \widetilde{F}\left(x^{\prime}, y_{n}, x_{n}\right)\right. \\
\left.-\sum_{j=0}^{N-1} \widetilde{F}_{\tilde{\xi}^{0}, j}\left(x^{\prime}, y_{n}, x_{n}\right) t^{-j / L}\right\} \longrightarrow \widetilde{F}_{\tilde{\xi}^{0}, N}\left(x^{\prime}, y_{n}, x_{n}\right), \\
\text { as } t \rightarrow \infty, \quad \text { in } \quad \mathscr{D}^{\prime}(X), \quad N=0,1,2, \ldots
\end{gathered}
$$

Moreover lie have 


$$
\begin{aligned}
& \cup_{j=0}^{\infty} \operatorname{supp} \widetilde{F}_{\xi^{0}, j}\left(x^{\prime}, y_{n}, x_{n}\right) \times\left\{\left(\xi^{0 \prime},-\xi_{n}^{0}, \xi_{n+1}^{0}\right)\right\} \\
& \subset W F\left(\tilde{F}\left(x^{\prime}, y_{n}, x_{n}\right)\right) \quad \text { for } \quad \tilde{\xi}^{0} \neq 0,
\end{aligned}
$$

and

$$
\overline{c h} \dagger\left[\cup_{j=0}^{\infty} \operatorname{supp} \tilde{F}_{\tilde{\xi}^{0}, j}\left(x^{\prime}, y_{n}, x_{n}\right)\right] \subset \tilde{K}_{\tilde{\xi}},
$$

where

$$
\widetilde{K}_{\tilde{\xi}^{0}}=\left\{\left(x^{\prime}, y_{n}, x_{n}\right) \in X ; x^{\prime} \cdot \eta^{\prime}-y_{n} \eta_{n}+x_{n} \eta_{n+1} \geqq 0 \quad \text { for all } \tilde{\eta} \in \Gamma_{\tilde{\xi}_{0}}\right\}
$$

and the closure in (2.18) is taken in $X$.

Remark. The inclusion of (2.18) can be replaced by the equality except in certain exceptional cases (see Example 5.1).

\section{Lopatinski Determinant}

The following lemma can be proved in the same way as in Theorem $3.1^{\prime}$ of Andersson [1] or in Lemma 5.1 of [2].

Lemma 3.1. Let $\xi^{0} \in \Xi^{n}$ and let $\tilde{M}$ be a compact set in $\Gamma\left(P_{\xi^{0}}, \vartheta\right)$. Then there exist a conic neighborhood $\tilde{\Delta}_{1}\left(\subset \Xi^{n}\right)$ of $\xi^{0}$ and positive numbers $C, t_{0}$ such that

$$
P\left(\xi-i t|\xi| \eta-i \gamma_{0} \vartheta\right) \neq 0 \quad \text { if } \quad \xi \in \tilde{\Delta_{1}},|\xi| \geqq C, \eta \in \tilde{M} \quad \text { and } \quad 0<t \leqq t_{0}
$$

This lemma can be also proved in the same way as in Lemma 3.7.

Lemma 3.2. Let $\xi^{0^{\prime}} \in \Xi^{n-1} \backslash\{0\}$ and let $M$ be a compact set in $\dot{\Gamma}_{\xi_{0} \circ}$. Then there exist a conic neighborhood $\Delta_{1}\left(\subset \Xi^{n-1}\right)$ of $\xi^{0 \prime}$ and positive numbers $C, t_{0}$ such that $P_{+}\left(\zeta^{\prime}, \lambda\right)$ is holomorphic in $\left(\zeta^{\prime}, \lambda\right)$ $\in \Lambda \times \mathbb{C}$, where

$$
\begin{array}{r}
\Lambda=\left\{\zeta^{\prime}=\xi^{\prime}-i t\left|\zeta^{\prime}\right| \eta^{\prime}-i \gamma_{0} \vartheta^{\prime} \in \Xi^{n-1}-i \Xi^{n-1} ; \xi^{\prime} \in \Delta_{1},\right. \\
\left.\left|\xi^{\prime}\right| \geqq C, \eta^{\prime} \in M \text { and } 0<t \leqq t_{0}\right\} .
\end{array}
$$

Therefore $R\left(\zeta^{\prime}\right)$ and $R_{j k}\left(\zeta^{\prime}\right)$ are also holomorphic in $\Lambda$. 
Proof. Write

$$
p_{j}\left(\zeta^{\prime}, \lambda\right)=\prod_{k=1}^{l_{j}^{+}}\left(\lambda-\lambda_{j k}^{+}\left(\zeta^{\prime}\right)\right) \Pi_{k=1}^{l_{j}^{-}}\left(\lambda-\lambda_{\bar{j} k}^{-}\left(\zeta^{\prime}\right)\right),
$$

where $\operatorname{Im} \lambda_{j k}^{ \pm}\left(\zeta^{\prime}\right) \gtrless 0$ for $\zeta^{\prime} \in \Xi^{n-1}-i \gamma_{0} \vartheta^{\prime}-i \Gamma_{0}$. It suffices to show that

$$
\left\{\lambda_{j k}^{+}\left(\zeta^{\prime}\right)\right\}_{k=1, \ldots, l_{j}^{+}} \cap\left\{\lambda_{j k}^{-}\left(\zeta^{\prime}\right)\right\}_{k=1, \ldots, l_{j}^{-}}=\emptyset \quad \text { for } \zeta^{\prime} \in \Lambda
$$

Put

$$
\mu_{j k}^{0}=\lim _{t \rightarrow \infty} t^{-1} \lambda_{j k}^{+}\left(t \xi^{0 \prime}\right) .
$$

If $\mu_{j h}^{0}$ is a real simple root or a non-real root of $p_{j}^{0}\left(\xi^{0^{\prime}}, \mu\right)=0$, it is obvious that

$$
\lambda_{j h}^{+}\left(\zeta^{\prime}\right) \neq \lambda_{j k}^{-}\left(\zeta^{\prime}\right), \quad 1 \leqq k \leqq l_{j}^{-}, \text {for } \zeta^{\prime} \in \Lambda
$$

when $C$ is large enough and $t_{0}$ is small enough. Thus we consider the case where $\mu_{j h}^{0}$ is a real multiple root of $p_{j}^{0}\left(\xi^{0 \prime}, \mu\right)=0$. Since $\dot{\Gamma}_{\xi^{\prime \prime}}$ $\times \Xi \subset \Gamma\left(p_{j\left(\xi^{0}, \mu_{j h}^{0}\right)}, \vartheta\right)$, to any compact set $\tilde{M}$ in $\dot{\Gamma}_{\xi^{0}} \times \Xi$ there exist a conic neighborhood $\tilde{\Delta}_{1}$ of $\left(\xi^{0 \prime}, \mu_{j h}^{0}\right)$ and positive numbers $C, t_{0}$ such that

$$
\begin{aligned}
& p_{j}\left(\xi^{\prime}-i t\left|\xi^{\prime}\right| \eta^{\prime}-i \gamma_{0} \vartheta^{\prime}, \lambda-i t\left|\xi^{\prime}\right| \eta_{n}\right) \neq 0 \\
& \quad \text { if } \quad\left(\xi^{\prime}, \lambda\right) \in \tilde{\Delta}_{1},\left|\xi^{\prime}\right| \geqq C, \eta \in \tilde{M} \quad \text { and } \quad 0<t \leqq t_{0}
\end{aligned}
$$

In fact, since $c\left|\xi^{\prime}\right| \geqq|\xi|$ for $\xi \in \tilde{\Delta}_{1}$, (3.4) is an immediate consequence of Lemma 3.1. Especially we put $\widetilde{M}=M \times\{0\}$. Then

$$
\begin{aligned}
& p_{j}\left(\xi^{\prime}-i t\left|\xi^{\prime}\right| \eta^{\prime}-i \gamma_{0} \vartheta^{\prime}, \lambda\right) \neq 0 \\
& \quad \text { if } \quad\left(\xi^{\prime}, \lambda\right) \in \tilde{\Delta}_{1},\left|\xi^{\prime}\right| \geqq C, \eta^{\prime} \in M \quad \text { and } \quad 0<t \leqq t_{0} .
\end{aligned}
$$

We may write

$$
\begin{gathered}
\tilde{\Delta}_{1}=\left\{\left(\xi^{\prime}, \lambda\right) \in \Xi^{n} \backslash\{0\} ;\left.|| \xi^{\prime}\right|^{-1} \xi^{\prime}-\left|\xi^{0^{\prime}}\right|^{-1} \xi^{0^{\prime}} \mid \leqq \varepsilon_{1},\right. \\
\\
\left.\left.|| \xi^{\prime}\right|^{-1} \lambda-\left|\xi^{0 \prime}\right|^{-1} \mu_{j h}^{0} \mid \leqq \varepsilon_{2}\right\} .
\end{gathered}
$$

On the other hand we have

$$
\left.|| \xi^{\prime}\right|^{-1} \lambda_{j h}^{+}\left(\xi^{\prime}-i t\left|\xi^{\prime}\right| \eta^{\prime}-i \gamma_{0} \vartheta^{\prime}\right)-\left|\xi^{0 \prime}\right|^{-1} \mu_{j h}^{0} \mid \leqq \varepsilon_{2}
$$


when $\left.|| \xi^{\prime}\right|^{-1} \xi^{\prime}-\left|\xi^{0}\right|^{-1} \xi^{0 \prime}\left|\leqq \varepsilon_{1},\right| \xi^{\prime} \mid \geqq C, \eta^{\prime} \in M$ and $0 \leqq t \leqq t_{0}$, if necessary, modifying $\varepsilon_{1}, C$ and $t_{0}$. If $\operatorname{Im} \lambda_{j h}^{+}\left(\xi^{\prime}-i t\left|\xi^{\prime}\right| \eta^{\prime}-i \gamma_{0} \vartheta^{\prime}\right)=0$ for $\xi^{\prime}-i t\left|\xi^{\prime}\right| \eta^{\prime}$ $-i \gamma_{0} \vartheta^{\prime} \in \Lambda$, then it follows from (3.6) that $\left(\xi^{\prime}, \lambda_{j h}^{+}\left(\xi^{\prime}-i t\left|\xi^{\prime}\right| \eta^{\prime}-i \gamma_{0} \vartheta^{\prime}\right)\right)$ $\in \tilde{\Delta_{1}}$. This contradicts (3.5). Thus we have $\operatorname{Im} \lambda_{j h}^{+}\left(\zeta^{\prime}\right) \neq 0$ for $\zeta^{\prime} \in \Lambda$. We can assume without loss of generality that $M$ is star-shaped with respect to $\vartheta^{\prime}$. Thus

$$
\operatorname{Im} \lambda_{j h}^{+}\left(\zeta^{\prime}\right)>0 \quad \text { when } \quad \zeta^{\prime} \text { in } \Lambda
$$

If $\lim _{t \rightarrow \infty} t^{-1} \lambda \bar{j}_{h^{\prime}}\left(t \xi^{0 \prime}\right)=\mu_{j h}^{0}$, by the same argument it is shown that

$$
\operatorname{Im} \lambda_{j h^{\prime}}\left(\zeta^{\prime}\right)<0 \quad \text { when } \zeta^{\prime} \text { in } \Lambda \text {. }
$$

Therefore we obtain (3.3).

Q.E.D.

Let $p(\xi)$ be a strictly hyperbolic polynomial with respect to $\vartheta$ and assume that $p^{0}(0,1) \neq 0$ and $p(\xi) \neq 0$ for $\xi \in \Xi^{n}-i \gamma_{0} \vartheta-i \Gamma$. Let $\xi^{0}$ be arbitrarily fixed in $\Xi^{n-1} \backslash\{0\}$ so that $p^{0}\left(\xi^{0 \prime}, \mu\right)=0$ has real multiple roots. Write

$$
\begin{aligned}
p\left(\xi^{\prime}, \lambda ; v\right) & =v^{m^{\prime}} p\left(v^{-1} \xi^{\prime}, v^{-1} \lambda\right) \\
& =p^{0}(\xi, \lambda)+v p^{1}(\xi, \lambda)+\cdots+v^{m^{\prime}} p^{m^{\prime}}
\end{aligned}
$$

where $\operatorname{deg} p=m^{\prime}$. We can assume without loss of generality that $\mu=0$ is an $l$-ple root of $p^{0}\left(\xi^{0 \prime}, \mu\right)=0$, where $l>1$. Thus Weierstrass' preparation theorem implies that

$$
\begin{array}{r}
p\left(\xi^{\prime}, \lambda ; v\right)=\left(\lambda^{l}+a_{1}\left(\xi^{\prime} ; v\right) \lambda^{l-1}+\cdots+a_{l}\left(\xi^{\prime} ; v\right)\right) q\left(\xi^{\prime}, \lambda ; v\right) \\
\text { for }\left|\xi^{\prime}-\xi^{0^{\prime}}\right|<\varepsilon \text { and }|v|<\varepsilon
\end{array}
$$

where the $a_{j}\left(\xi^{\prime} ; v\right)$ and $q\left(\xi^{\prime}, \lambda ; v\right)$ are holomorphic for $\left|\xi^{\prime}-\xi^{0^{\prime}}\right|<\varepsilon$ and $|v|<\varepsilon$ and $a_{j}\left(\xi^{0} ; 0\right)=0$ and $q\left(\xi^{\prime}, \lambda ; v\right) \neq 0$ for $\left|\xi^{\prime}-\xi^{0^{\prime}}\right|<\varepsilon,|v|<\varepsilon$ and $|\lambda|<\varepsilon$. Then

$$
a_{j}\left(\zeta^{\prime} ; v\right)=\sum_{k+|\alpha| \geq 1} a_{j k \alpha} v^{k}\left(\xi^{\prime}-\xi^{0 \prime}\right)^{\alpha}, \quad|v|<\varepsilon,\left|\xi^{\prime}-\xi^{0 \prime}\right|<\varepsilon
$$

and, therefore,

$$
a_{J}\left(\xi^{0^{\prime}}+v \eta^{\prime} ; v\right)=\sum_{k=1}^{\infty} a_{j k}\left(\eta^{\prime}\right) v^{k}
$$


where

$$
a_{j k}\left(\eta^{\prime}\right)=\sum_{i+|\alpha|=k} a_{j i \alpha} \eta^{\prime \alpha}
$$

It is easy to see that

$$
a_{l 1}\left(\eta^{\prime}\right)=\text { const. } p_{\left(\xi^{0 \prime}, 0\right)}(\eta)
$$

Let $\zeta\left(\xi^{\prime} ; v\right)$ be a root of $p\left(\xi^{\prime}, \lambda ; v\right)=0$ such that $\zeta\left(\xi^{0 \prime} ; 0\right)=0$. We modify Lemma 2.5 in [9] and obtain the following

Lemma 3.3. For any compact set $K$ in $\Xi^{n-1}-i \Gamma_{1}$, where $\Gamma_{1} \times \Xi$ $=\Gamma\left(p_{\left(\xi^{0}, 0\right)}, \vartheta\right)$, any positive integer $N_{1}$ and any non-negative integer $\mathrm{N}_{2}$ there exist positive numbers $v_{0}, r_{0}$ and $C$ such that

$$
\begin{aligned}
& \zeta\left(\xi^{0 \prime}+v \eta^{\prime} ; v r^{-1}\right)=\sum_{j=1}^{N_{1}} c_{j}\left(r \eta^{\prime}\right)\left(v r^{-1}\right)^{j / l} \\
& \quad+v^{\left(N_{1}+1\right) / l}\left(\sum_{j=0}^{N_{2}} d_{j}\left(\eta^{\prime} ; v\right) r^{-j}+O\left(r^{-N_{2}-1}\right)\right)
\end{aligned}
$$

and $\left|d_{j}\left(\eta^{\prime} ; v\right)\right|<C, 0 \leqq j \leqq N_{2}$, if $r_{0} \eta^{\prime} \in \Xi^{n-1}-i \gamma_{0} \vartheta^{\prime}-i \Gamma_{1}, \alpha \eta^{\prime} \in K$ for some $\alpha \in \mathbf{C}(|\alpha|=1), 0 \leqq \nu \leqq v_{0}$ and $r \geqq r_{0}$.

\section{Proof. $\dagger \quad$ Write}

$$
\zeta\left(\xi^{0^{\prime}}+v \eta^{\prime} ; v r^{-1}\right)=\sum_{j=1}^{N_{1}} c_{j}\left(r \eta^{\prime}\right)\left(v r^{-1}\right)^{j / l}+\zeta_{N_{1}+1}\left(r \eta^{\prime} ; v r^{-1}\right)
$$

(see Lemma 2.5 in [9]). Then we have

$$
c_{1}\left(r \eta^{\prime}\right)=\left\{-a_{l 1}\left(r \eta^{\prime}\right)\right\}^{1 / l}
$$

and, inductively, determine the $c_{j}\left(r \eta^{\prime}\right)$ by the equations

$$
\begin{aligned}
& l c_{1}\left(r \eta^{\prime}\right)^{l-1} c_{j}\left(r \eta^{\prime}\right)+\sum_{\substack{1 \leqq j_{1}, \ldots, j_{l} \leqq j-1 \\
j_{1}+\cdots+j_{l}=l-1+j}} c_{j_{1}}\left(r \eta^{\prime}\right) \cdots c_{j_{l}}\left(r \eta^{\prime}\right) \\
& \quad+\sum_{k-0}^{l-1} \sum_{\substack{1 \leqq j_{1}, \ldots, j_{k} \leqq j-1 \\
l l+j_{1}+\cdots+j_{k}=l-1+j}} c_{j_{1}}\left(r \eta^{\prime}\right) \cdots c_{j_{k}}\left(r \eta^{\prime}\right) a_{l-k, h}\left(r \eta^{\prime}\right)=0 .
\end{aligned}
$$

In fact, we have only to note that $\left|c_{1}\left(r \eta^{\prime}\right)\right| \geqq C r^{1 / l}$ if $\alpha \eta^{\prime} \in K$ for some $\alpha \in \mathbf{C}(|\alpha|=1)$ and $r \geqq r_{0}$, where $r_{0}$ is large enough. $\zeta_{N_{1}+1}\left(r \eta^{\prime} ; v r^{-1}\right)$ satisfies the equation

$\dagger$ The same argument as in the footnote of Lemma 2.5 in [9] gives a simpler proof of this lemma. Then Lemma 3.5 will become obvious. 


$$
\begin{aligned}
& \sum_{k=0}^{l-1}\left(\begin{array}{l}
l \\
k
\end{array}\right)\left\{c_{1}\left(r \eta^{\prime}\right)^{k}\left(v r^{-1}\right)^{k / l}+\sum_{\substack{1 \leqq j_{1}, \ldots, j_{k} \leqq N_{1} \\
j_{1}+\ldots+j_{k} \geqq k+1}}\right. \\
& \left.c_{j_{1}}\left(r \eta^{\prime}\right) \cdots c_{j_{k}}\left(r \eta^{\prime}\right)\left(v r^{-1}\right)^{\left(j_{1}+\cdots+j_{k}\right) / l}\right\} \zeta_{N_{1}+1}\left(r \eta^{\prime} ; v r^{-1}\right)^{l-k} \\
& +\sum_{\substack{1 \leq j_{1}, \ldots, j_{l} \leqq N_{1} \\
j_{1}+\cdots+j_{l} \geqq l+N_{1}}} c_{j_{1}}\left(r \eta^{\prime}\right) \cdots c_{j_{l}}\left(r \eta^{\prime}\right)\left(v r^{-1}\right)^{\left(j_{1}+\cdots+j_{l}\right) / l} \\
& +\sum_{h=1}^{l-1} \sum_{k=0}^{h-1} \sum_{1 \leqq j_{1}, \ldots, j_{k} \leqq N_{1}} \sum_{q=1}^{\infty}\left(\begin{array}{c}
h \\
k
\end{array}\right) c_{j_{1}}\left(r \eta^{\prime}\right) \cdots c_{j_{k}}\left(r \eta^{\prime}\right) \\
& \times a_{l-h, q}\left(r \eta^{\prime}\right)\left(v r^{-1}\right)^{\left(j_{1}+\cdots+j_{k}\right) / l+q} \zeta_{N_{1}+1}\left(r \eta^{\prime} ; v r^{-1}\right)^{h-k} \\
& +\sum_{h=0}^{l-1} \sum_{\substack{1 \leq j_{1}, \ldots, j_{h} \leqq N_{1} \\
j 1+\ldots+j_{h}+l q}} c_{j_{1} \geqq l+N}\left(r \eta^{\prime}\right) \ldots c_{j_{h}}\left(r \eta^{\prime}\right) a_{l-h, q}\left(r \eta^{\prime}\right) \\
& \times\left(v r^{-1}\right)^{\left(j_{1}+\cdots+j_{h}\right) / l+q}=0 .
\end{aligned}
$$

Since

$$
c_{j}\left(r \eta^{\prime}\right)=r^{j / l} \sum_{k=0}^{\infty} c_{j k}\left(\eta^{\prime}\right) r^{-k}, \quad c_{10}\left(\eta^{\prime}\right) \neq 0
$$

when $r_{0} \eta^{\prime} \in \Xi^{n-1}-i \gamma_{0} \vartheta^{\prime}-i \Gamma_{1}, \alpha \eta^{\prime} \in K$ for some $\alpha \in \mathbb{C}(|\alpha|=1)$ and $r \geq r_{0}$, whose convergence is uniform, (3.14) can be written in the form

$$
\begin{aligned}
& \sum_{k=0}^{l-1} \sum_{q=0}^{M}\left\{b_{k q}\left(\eta^{\prime} ; v\right) r^{-q}+O\left(r^{-M-1}\right)\right\} v^{k / l} \zeta_{N_{1}+1}\left(r \eta^{\prime} ; v r^{-1}\right)^{l-k} \\
& \quad+v^{N_{1} / l+1}\left\{\sum_{q=0}^{N_{2}} e_{q}\left(\eta^{\prime} ; v\right) r^{-q}+O\left(r^{-N_{2}-1}\right)\right\}=0
\end{aligned}
$$

where $M$ is a non-negative integer and

$$
b_{k 0}\left(\eta^{\prime} ; v\right)=\left(\begin{array}{l}
l \\
k
\end{array}\right) c_{10}\left(\eta^{\prime}\right)^{k}+O\left(v^{1 / l}\right)
$$

By the same argument as in Lemma 2.5 of [9] it can be easily proved that

$$
\left|\zeta_{N_{1}+1}\left(r \eta^{\prime} ; v r^{-1}\right)\right| \leqq C v^{\left(N_{1}+1\right) / l}
$$

Thus if $v_{0}$ is small enough, the $d_{j}\left(\eta^{\prime} ; v\right)$ are determined by the equations

$$
\begin{aligned}
& \sum_{k=1}^{l} v^{(k-1) N_{1} / l} b_{l-k, 0}\left(\eta^{\prime} ; v\right) d_{0}\left(\eta^{\prime} ; v\right)^{k}+e_{0}\left(\eta^{\prime} ; v\right)=0, \\
& \sum_{k=1}^{l} k v^{(k-1) N_{1} / l} b_{l-k, 0}\left(\eta^{\prime} ; v\right) d_{0}\left(\eta^{\prime} ; v\right)^{k-1} d_{j}\left(\eta^{\prime} ; v\right)
\end{aligned}
$$




$$
\begin{aligned}
& +\sum_{k=1}^{l} \sum_{\substack{0 \leqq j_{1}, \ldots, j_{k}<j \\
q+j_{1}+\cdots+j_{k}=j}} v^{(k-1) N_{1} / l} b_{l-k, q}\left(\eta^{\prime} ; v\right) \\
& \times d_{j_{1}}\left(\eta^{\prime} ; v\right) \cdots d_{j_{k}}\left(\eta^{\prime} ; v\right)+e_{j}\left(\eta^{\prime} ; v\right)=0 .
\end{aligned}
$$

In fact, we have only to note that (3.16) has a unique solution $d_{0}\left(\eta^{\prime} ; v\right)$ such that $d_{0}\left(\eta^{\prime} ; v\right)=O(1)$ as $v \rightarrow 0$, and that

$$
\left|\sum_{k=1}^{l} k v^{(k-1) N_{1} / l} b_{l-k, 0}\left(\eta^{\prime} ; v\right) d_{0}\left(\eta^{\prime} ; v\right)^{k-1}\right| \geqq C .
$$

Put

$$
\zeta_{N_{1}+1}\left(r \eta^{\prime} ; v r^{-1}\right)=v^{\left(N_{1}+1\right) / l}\left\{\sum_{j=0}^{N_{2}} d_{j}\left(\eta^{\prime} ; v\right) r^{-j}+\tilde{\zeta}_{N_{1}, N_{2}}\left(\eta^{\prime} ; r, v\right)\right\}
$$

Then $\tilde{\zeta}_{N_{1}, N_{2}}\left(\eta^{\prime} ; r, v\right)$ satisfies the equation

$$
\begin{aligned}
& \sum_{h=1}^{l}\left\{\sum _ { k = h } ^ { l } ( \begin{array} { l } 
{ k } \\
{ h }
\end{array} ) v ^ { ( k - 1 ) N _ { 1 } / l } \left(b_{l-k, 0}\left(\eta^{\prime} ; v\right) d_{0}\left(\eta^{\prime} ; v\right)^{k-h}\right.\right. \\
& \left.\left.+O\left(r^{-1}\right)\right)\right\} \tilde{\zeta}_{N_{1}, N_{2}}\left(\eta^{\prime} ; r, v\right)^{h}+O\left(r^{-N_{2}-1}\right)=0 .
\end{aligned}
$$

Since $\tilde{\zeta}_{N_{1}, N_{2}}\left(\eta^{\prime} ; r, v\right)=O(1)$ as $v \rightarrow 0$, we have

$$
\left|\tilde{\zeta}_{N_{1}, N_{2}}\left(\eta^{\prime} ; r, v\right)\right| \leqq C r^{-N_{2}-1}
$$

when $r_{0} \eta^{\prime} \in \Xi^{n-1}-i \gamma_{0} \vartheta^{\prime}-i \Gamma_{1}, \alpha \eta^{\prime} \in K$ for some $\alpha \in \mathbb{C}(|\alpha|=1), 0 \leqq v \leqq v_{0}$ and $r \geqq r_{0}$.

Q.E.D.

Since $\lambda=v^{-1} r \zeta\left(\xi^{0 \prime}+v \eta^{\prime} ; v r^{-1}\right)$ is a root of $p\left(v^{-1} r \xi^{0^{\prime}}+r \eta^{\prime}, \lambda\right)=0$, from Lemma 3.3 the following lemma can be easily proved.

Lemma 3.4. Let $K$ be a compact set in $\Xi^{n-1}-i \dot{\Gamma}_{\xi^{0}}$. For any non-negative integer $N$ there exist positive numbers $v_{0}$ and $r_{0}$ such that

$$
\begin{gathered}
v^{h_{1}} R\left(v^{-1} r \xi^{0^{\prime}}+r \eta^{\prime}\right)=r^{h_{1}} \sum_{j=0}^{N} Q_{j}\left(r \eta^{\prime}\right)\left(v r^{-1}\right)^{j / L} \\
+O\left(r^{h_{0}} v^{(N+1) / L}\right)
\end{gathered}
$$

if $\quad r_{0} \eta^{\prime} \in \Xi^{n-1}-i \gamma_{0} \vartheta^{\prime}-i \dot{\Gamma}_{\xi^{0}}, \quad \alpha \eta^{\prime} \in K$ for some $\alpha \in \mathbf{C}(|\alpha|=1), \quad 0<\nu \leqq v_{0}$ and $r \geqq r_{0}$, where $h_{0}$ and $h_{1}$ were defined by (2.2) and (2.8), respectively, and $L$ is a positive integer.

Define the principal parts $Q_{j}^{0}$ of $Q_{j}$ and rational numbers $q_{j}$ by 


$$
Q_{j}\left(r \eta^{\prime}\right)=r^{q_{j}}\left\{Q_{j}^{0}\left(\eta^{\prime}\right)+r^{-1} Q_{j}^{1}\left(\eta^{\prime}\right)+\cdots\right\}, \quad Q_{j}^{0}\left(\eta^{\prime}\right) \not \equiv 0 .
$$

Here it follows from Lemma 3.3 and (3.15) that the developments are meaningful when $r_{0} \eta^{\prime} \in \Xi^{n-1}-i \gamma_{0} \vartheta^{\prime}-i \dot{\Gamma}_{\xi^{0}}, \alpha \eta^{\prime} \in K$ for some $\alpha \in \mathbb{C}$ $(|\alpha|=1)$ and $r \leqq r_{0}$, where $K$ is a compact set in $\Xi^{n-1}-i \dot{\Gamma}_{\xi^{0}}$, and $r_{0}$ is large enough. Moreover it is easy to see that $p_{j} \equiv h_{1}+q_{j}-j / L$ is an integer and that $p_{j} \leqq h_{0}$. Put

$$
p=\max p_{j}, \quad \alpha=\min _{p=p_{j}} j .
$$

Lemma 3.5. $p=h_{0}$.

Proof. It is obvious that $p \leqq h_{0}$. Let us assume $p \supsetneqq h_{0}$. From Lemma 3.4 we have

$$
\begin{aligned}
& \lim _{v \rightarrow 0} v^{-N} \widetilde{R}_{0}\left(v^{-1} \xi^{0 \prime}-i \vartheta^{\prime}\right) \\
& \quad=\lim _{v \rightarrow 0} \lim _{r \rightarrow \infty} v^{-N} r^{-h_{0}} R\left(v^{-1} r \xi^{0 \prime}-i r \vartheta^{\prime}\right)=0
\end{aligned}
$$

for any integer $N$.

Put

$$
\phi(r, v)=\left|r^{-h_{0}} R\left(v^{-1} r \xi^{0 \prime}-i r \vartheta^{\prime}\right)\right|, \quad r \geqq r_{0}, \quad 0<v \leqq v_{0},
$$

where $r_{0}>\gamma_{1}$. By Seidenberg's lemma we see that

$$
\phi_{\min }(v) \equiv \inf _{r \geq r_{0}} \phi(r, v)=C_{\beta} v^{\beta}(1+o(1)) \quad \text { as } \quad v \rightarrow 0 .
$$

Since $r^{-h_{0}} R\left(v^{-1} r \xi^{0 \prime}-i r \vartheta^{\prime}\right) \neq 0$ and $r^{-h_{0}} R\left(v^{-1} r \xi^{0 \prime}-i r \vartheta^{\prime}\right) \stackrel{r \rightarrow \infty}{\longrightarrow} \widetilde{R}_{0}\left(v^{-1} \xi^{0 \prime}-i \vartheta^{\prime}\right)$ $\neq 0$, we obtain $C_{\beta} \neq 0(>0)$. Thus

$$
\left|\widetilde{R}_{0}\left(v^{-1} \xi^{0^{\prime}}-i \vartheta^{\prime}\right)\right| \geqq C_{\beta} \nu^{\beta}(1+o(1)) \quad \text { as } \quad v \rightarrow 0 .
$$

This is a contradiction to (3.20).

Q.E.D.

Lemma 3.6. There exists the localization $\widetilde{R}_{0 \xi^{\circ}}\left(\eta^{\prime}\right)$ of $\widetilde{R}_{0}\left(\xi^{\prime}\right)$ at $\xi^{0 \prime}$ and

$$
\widetilde{R}_{0 \xi^{0}}\left(\eta^{\prime}\right)=Q_{\alpha}^{0}\left(\eta^{\prime}\right),
$$

i.e. 


$$
\begin{array}{r}
\widetilde{R}_{0}\left(v^{-1} \xi^{0 \prime}+\eta^{\prime}\right)=v^{-h_{1}+\alpha / L}\left(\widetilde{R}_{0 \xi^{0}}\left(\eta^{\prime}\right)+o(1)\right) \quad \text { as } \quad \nu \rightarrow 0, \\
\text { for each } \eta^{\prime} \in \Xi^{n-1}-i \dot{\Gamma} .
\end{array}
$$

Remark. $\widetilde{R}_{0 \xi^{0}}\left(\eta^{\prime}\right)$ is holomorphic in $\Xi^{n-1}-i \dot{\Gamma}_{\xi^{0}}$

Proof.

$$
\begin{aligned}
& \lim \sup _{r \rightarrow \infty}\left|r^{-h_{0}} R\left(v^{-1} r \xi^{0 \prime}+r \eta^{\prime}\right)-v^{-h_{1}+\alpha / L} Q_{\alpha}^{0}\left(\eta^{\prime}\right)\right| \\
& \leqq C_{\eta^{\prime}} v^{(\alpha+1) / L-h_{1}}, \quad \eta^{\prime} \in \Xi^{n-1}-i \dot{\Gamma}_{\xi^{0}}
\end{aligned}
$$

Thus

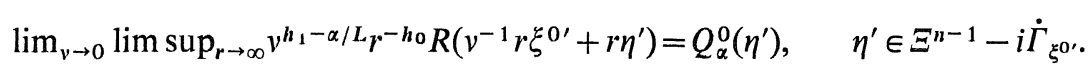

On the other hand

$$
\lim \sup _{r \rightarrow \infty} r^{-h_{0}} R\left(v^{-1} r \xi^{0^{\prime}}+r \eta^{\prime}\right)=\widetilde{R}_{0}\left(v^{-1} \xi^{0^{\prime}}+\eta^{\prime}\right), \quad \eta^{\prime} \in \Xi^{n-1}-i \ddot{\Gamma} .
$$

This proves the lemma.

Q.E.D.

Let $\dot{\Sigma}_{\xi^{0}}^{0}$, be the component of the set $\left\{\eta^{\prime} \in \dot{\Gamma}_{\xi^{0}} ; \widetilde{R}_{0 \xi^{0}}\left(-i \eta^{\prime}\right) \neq 0\right\}$ which contains $\vartheta^{\prime}$. Here we define $\dot{\Sigma}_{\xi^{\prime}}^{0}=\dot{\Sigma}$ if $\xi^{0^{\prime}}=0$. In fact, by the same argument as in Lemma 3.1 of [9] it follows that

$$
\tilde{R}_{0 \xi^{0}},\left(\eta^{\prime}\right) \neq 0 \quad \text { for } \quad \eta^{\prime} \in \Xi^{n-1}-i \dot{\Sigma} \text {. }
$$

Especially we obtain $\widetilde{R}_{0 \xi^{0}}\left(-i \vartheta^{\prime}\right) \neq 0$. We can also prove that $\ddot{\Sigma}_{\xi^{0}}^{0}$, is an open convex cone and that $\widetilde{R}_{0 \xi^{0}},\left(\eta^{\prime}\right) \neq 0$ for $\eta^{\prime} \in \Xi^{n-1}-i \dot{\Sigma}_{\xi^{0}}$.

In the proof of Theorem 4.2 the following lemma plays a key role.

Lemma 3.7. For any compact set $M$ in $\dot{\Sigma}_{\xi 0}^{0}$, there exist a conic neighborhood $\Delta_{1}\left(\subset \Xi^{n-1}\right)$ of $\xi^{0^{\prime}}$ and positive numbers $C, t_{0}$ such that

$$
\begin{array}{r}
R\left(\xi^{\prime}-i t\left|\xi^{\prime}\right| \eta^{\prime}-i \gamma_{1} \vartheta^{\prime}\right) \neq 0 \quad \text { if } \quad \eta^{\prime} \in M, \xi^{\prime} \in \Delta_{1},\left|\xi^{\prime}\right| \geqq C \quad \text { and } \\
0<t \leqq t_{0}
\end{array}
$$

Proof. Put

$$
f\left(v, r, \zeta^{\prime} . s, t, \eta^{\prime}\right)=R\left(v^{-1} r \xi^{0 \prime}+r \zeta^{\prime}-i t r \eta^{\prime}-i s \vartheta^{\prime}-i \gamma_{1} \vartheta^{\prime}\right),
$$


where $0<v \leqq v_{0}, \quad r \geqq r_{0}, \quad \zeta^{\prime} \in \Xi^{n-1}, \quad\left|\zeta^{\prime}\right| \leqq \varepsilon, \quad \operatorname{Re} s \geqq 0, \quad \operatorname{Re} t \geqq 0, \quad \operatorname{Re}(s+t)>0$, $|s| \leqq s_{0},|t| \leqq t_{0}$ and $\eta^{\prime} \in M$. Here positive numbers $v_{0}, r_{0}, \varepsilon, s_{0}$ and $t_{0}$ are chosen so that Lemma 3.2 is applicable and $f\left(v, r, \zeta^{\prime}, s, t, \eta^{\prime}\right)$ is well-defined, continuous in all variables and holomorphic in $t, \operatorname{Re} t>0$. (1.7) implies that

$$
f\left(v, r, \zeta^{\prime}, s, t, \eta^{\prime}\right) \neq 0 \quad \text { when } \quad \operatorname{Re} s>0 \text { and } \operatorname{Re} t=0 \text {. }
$$

Moreover we have

$$
f\left(v, r, \zeta^{\prime}, s, t, \eta^{\prime}\right) \neq 0 \quad \text { for } \quad v=v_{0}, \operatorname{Re} s \geqq 0, \operatorname{Re} t>0 \text { and }|t|=t_{0}
$$

if necessary, modifying $v_{0}, \varepsilon$ and $s_{0}$. In fact, there exists a compact set $K$ in $\Xi^{n-1}-i \dot{\Sigma}_{\xi^{0}}^{0}$, such that $\bar{t}|t|^{-1}\left(\zeta^{\prime}-i t \eta^{\prime}-i r^{-1}\left(s+\gamma_{1}\right) \vartheta^{\prime}\right) \in K$ when $r \geqq r_{0},\left|\zeta^{\prime}\right| \leqq \varepsilon,|s| \leqq s_{0},|t|=t_{0}$ and $\eta^{\prime} \in M$. Thus it follows from Lemma 3.4 that

$$
\begin{gathered}
f\left(v_{0}, r, \zeta^{\prime}, s, t, \eta^{\prime}\right)=v_{0}^{-h_{1}}\left\{\sum_{j=0}^{\alpha} Q_{j}^{0}\left(\zeta^{\prime}-i t \eta^{\prime}-i r^{-1}\left(s+\gamma_{1}\right) \vartheta^{\prime}\right)\right. \\
\left.\times v_{0}^{j / L} r^{p_{j}}+\sum_{j=0}^{\alpha} \mathrm{O}\left(v_{0}^{j / L} r^{p_{j}-1}\right)+\mathrm{O}\left(v_{0}^{(\alpha+1) / L} r^{h_{0}}\right)\right\} .
\end{gathered}
$$

From homogeneity of $Q_{\alpha}^{0}\left(\eta^{\prime}\right)$ there exists $\delta>0$ such that

$$
\left|Q_{\alpha}^{0}\left(\zeta^{\prime}-i t \eta^{\prime}-i r^{-1}\left(s+\gamma_{1}\right) \vartheta^{\prime}\right)\right|>\delta
$$

if $r \geqq r_{0},\left|\zeta^{\prime}\right| \leqq \varepsilon,|s| \leqq s_{0},|t|=t_{0} \quad$ and $\quad \eta^{\prime} \in M$. Since $p_{\alpha}=h_{0}$ and $p_{j}<h_{0}$ for $0 \leqq j \leqq \alpha-1$, (3.27) and (3.28) imply (3.26). Now we assume that there exist $r_{1}\left(\geqq r_{0}\right), \zeta^{1^{\prime}} \in \Xi^{n-1}, s_{1}, t_{1} \in \mathbb{C}$ and $\eta^{1^{\prime}} \in M$ such that $\left|\zeta^{1^{\prime}}\right| \leqq \varepsilon$, $\operatorname{Re} s_{1} \geqq 0,\left|s_{1}\right| \leqq s_{0}, \operatorname{Re} t_{1}>0$ and $\left|t_{1}\right| \leqq t_{0}$ and $f\left(v_{0}, r_{1}, \zeta^{1 \prime}, s_{1}, t_{1}, \eta^{1 \prime}\right)=0$. Then we can assume that $\operatorname{Re} s_{1}>0$. There exists a continuous function $t(\theta)$ defined on $\left[0, \theta_{0}\right), 0<\theta_{0} \leqq 1$, such that

$$
f\left(v_{0}, r_{1}, \zeta^{1^{\prime}}, s_{1}, t(\theta),(1-\theta) \eta^{1^{\prime}}+\theta \vartheta^{\prime}\right)=0, \quad t(0)=t_{1} \text {. }
$$

Since (3.25) and (3.26) imply that $|t(\theta)| \neq t_{0}$ and $\operatorname{Re} t(\theta) \neq 0$, it follows that $|t(\theta)|<t_{0}$ and $\operatorname{Re} t(\theta)>0$. Thus $t(\theta)$ can be defined on $[0,1]$. This is a contradiction to $f\left(v_{0}, r_{1}, \zeta^{1}, s_{1}, t, \vartheta^{\prime}\right) \neq 0$ for $\operatorname{Re} t \geqq 0$. Therefore

$$
R\left(v_{0}^{-1} r \xi^{0 \prime}+r \zeta^{\prime}-i t r \eta^{\prime}-i s \vartheta^{\prime}-i \gamma_{1} \vartheta^{\prime}\right) \neq 0
$$

when $r \geqq r_{0}, \quad\left|\zeta^{\prime}\right| \leqq \varepsilon, \quad \operatorname{Re} s \geqq 0,|s| \leqq s_{0}, \quad \operatorname{Re} t>0,|t| \leqq t_{0}$ and $\eta^{\prime} \in M$. This 
proves the lemma.

Q.E.D.

Lemma 3.8. $\dot{\Sigma}_{\xi}^{0}, \subset \dot{\Sigma}_{\xi 0}$.

Remark. In $\S 5$ we shall give an example such that $\dot{\Sigma}_{\xi^{\circ}}^{0} \varsubsetneqq \dot{\Sigma}_{\xi^{\prime}}$.

Proof. By the same argument as in the proof of Lemma 3.7 we have

$$
R\left(v^{-1} r \xi^{0 \prime}+\zeta^{\prime}-i \eta^{\prime}-i \gamma_{1} \vartheta^{\prime}\right)=f\left(v, r, r^{-1} \zeta^{\prime}, 0, r^{-1}, \eta^{\prime}\right) \neq 0
$$

when $\eta^{\prime} \in M \subset \dot{\Sigma}_{\xi^{\circ}}^{0},\left|\zeta^{\prime}\right| \leqq \varepsilon r, 0<v \leqq v_{0}, r \geqq r_{0}$ and $r>v^{-N}$, where $N$ is large enough. Now assume that there exists $\eta^{\prime} \in \dot{\Sigma}_{\xi_{0}^{0}}^{0}$ such that $Q_{0}^{0}\left(-i \eta^{\prime}\right)$ $=0$. Then there exist $\zeta^{0^{\prime}}$ and $\delta>0$ such that $-i \eta^{\prime}+\mu \zeta^{0^{\prime}} \in \Xi^{n-1}-i \dot{\Sigma}_{\xi^{0}}^{0}$, and $Q_{0}^{0}\left(-i \eta^{\prime}+\mu \zeta^{0 \prime}\right) \neq 0$ for $|\mu|=\delta$. On the other hand

$$
\begin{aligned}
& \left|Q_{0}\left(-i t\left(\eta^{\prime}+\mu \zeta^{0 \prime}\right)\right)-Q_{0}^{0}\left(-i t\left(\eta^{\prime}+\mu \zeta^{0^{\prime}}\right)\right)\right| \\
& \quad \leqq \frac{1}{2}\left|Q_{0}^{0}\left(-i t\left(\eta^{\prime}+\mu \zeta^{0 \prime}\right)\right)\right| \quad \text { for }|\mu|=\delta \text { and } t>T,
\end{aligned}
$$

where $T$ is large enough. Thus Rouché's theorem implies that for fixed $t(>T)$ there exists $\mu_{0} \in \mathbb{C}$ such that $\left|\mu_{0}\right|<\delta$ and $Q_{0}\left(-i t\left(\eta^{\prime}+\right.\right.$ $\left.\left.\mu_{0} \zeta^{0 \prime}\right)\right)=0$. Therefore there exists $\zeta^{\prime} \in \Xi^{n-1}-i \gamma_{1} \vartheta^{\prime}-i \dot{\Sigma}_{\xi^{0}}^{0}$, such that $Q_{0}\left(\zeta^{\prime}\right)=0$. From $Q_{0}\left(\xi^{\prime}\right) \neq \equiv$ it follows that there exist $\eta^{0^{\prime}}$ and $\delta>0$ such that $\zeta^{\prime}+\mu \eta^{0 \prime} \in \Xi^{n-1}-i \gamma_{1} \vartheta^{\prime}-i \dot{\Sigma}_{\xi^{0}}^{0}$ and $Q_{0}\left(\zeta^{\prime}+\mu \eta^{0 \prime}\right) \neq 0$ for $|\mu|=\delta$. On the other hand Lemma 2.4 implies that

$$
\begin{aligned}
& \left|\left(v^{-1} r\right)^{h_{1}} R\left(v^{-1} r \xi^{0^{\prime}}+\zeta^{\prime}+\mu \eta^{0 \prime}\right)-Q_{0}\left(\zeta^{\prime}+\mu \eta^{0 \prime}\right)\right| \\
& \leqq \frac{1}{2}\left|Q_{0}\left(\zeta^{\prime}+\mu \eta^{0 \prime}\right)\right| \quad \text { for }|\mu|=\delta \text { and } v^{-1} r>T,
\end{aligned}
$$

where $T$ is large enough. Thus Rouché's theorem leads us to a contradiction to (3.29). This proves the lemma.

Q.E.D.

\section{Wave Front Set}

First one observes that there exist bounded sequences $\left\{\phi_{N}\right\}$ in $C_{0}^{\infty}(X)$ such that $\phi_{N}=1$ on a fixed neighborhood of $\left(x^{0}, y_{n}^{0}, x_{n}^{0}\right)$ in $X$, independent of $N$, and 


$$
\left|\widetilde{D}^{\alpha} \phi_{N}\right| \leqq C(C N)^{|\alpha|} \quad \text { for } \quad|\alpha| \leqq N
$$

where $\tilde{D}=i^{-1}\left(\partial / \partial x_{1}, \ldots, \partial / \partial x_{n-1}, \partial / \partial y_{n}, \partial / \partial x_{n}\right)$ (see [5]).

Definition 4.1. Let $u\left(x^{\prime}, y_{n}, x_{n}\right) \in \mathscr{D}^{\prime}(X)$. Then the analytic wave front set $W F_{A}(u)$ of $u$ is defined as the complement, in $X \times\left(\Xi^{n+1} \backslash\{0\}\right)$, of the points $\left(\left(x^{0 \prime}, y_{n}^{0}, x_{n}^{0}\right), \tilde{\xi}^{0}\right)$ such that for some sequence $\left\{\phi_{N}\right\}$ of the above type there exists a conic neighborhood $\Delta$ of $\tilde{\xi}^{0}$ in $\Xi^{n+1} \backslash\{0\}$ with

$$
\left|\mathscr{F}_{\left(x^{\prime}, y_{n}, x_{n}\right)}\left[\phi_{N} u\right](\tilde{\xi})\right| \leqq C(C N)^{N}(1+|\tilde{\xi}|)^{-N} \quad \text { when } \quad \tilde{\xi} \in \Delta
$$

Define

$$
\Gamma_{\tilde{\xi}^{0}}^{0}=\left(\Gamma\left(P_{\xi^{0}}, \vartheta\right) \times \Xi\right) \cap \widetilde{\Gamma}_{\left(\xi^{0}, \xi_{n+1}^{0}\right)} \cap\left(\dot{\Sigma}_{\xi^{0}}^{0}, \times \Xi^{2}\right),
$$

(4.4) $\tilde{K}_{\xi_{0}^{0}}^{0}=\left\{\left(x^{\prime}, y_{n}, x_{n}\right) \in X ; x^{\prime} \cdot \eta^{\prime}-y_{n} \eta_{n}+x_{n} \eta_{n+1} \geqq 0 \quad\right.$ for all $\left.\tilde{\eta} \in \Gamma_{\tilde{\xi}}^{0}\right\}$, where $\tilde{\xi}^{0} \in \Xi^{n+1} \backslash\{0\}$ and $\widetilde{K}_{\xi_{0}^{0}}^{0}=\varnothing$ for $\xi^{0 \prime}=0$. Then our main theorem is stated as follows.

Theorem 4.2. Under the conditions (A.1) and (A.2)

$$
(W F(\widetilde{F}) \subset) W F_{A}\left(\widetilde{F}\left(x^{\prime}, y_{n}, x_{n}\right)\right) \subset \cup_{\tilde{\xi} \in \tilde{z}^{n+1} \mid\{0\}} \tilde{K}_{\xi}^{0} \times\left\{\left(\xi^{\prime},-\xi_{n}, \xi_{n+1}\right)\right\}
$$

Remark.

$$
\begin{aligned}
& \cup_{\tilde{\xi} \in \Xi^{n+1} \backslash\{0\}} \cup_{j=0}^{\infty} \operatorname{supp} \widetilde{F}_{\tilde{\xi}, j}\left(x^{\prime}, y_{n}, x_{n}\right) \subset \operatorname{sing} \operatorname{supp} \tilde{F}\left(x^{\prime}, y_{n}, x_{n}\right) \\
& \subset \text { anal sing } \operatorname{supp} \widetilde{F}\left(x^{\prime}, y_{n}, x_{n}\right) \subset \cup_{\tilde{\xi}_{E} \in \Xi^{n+1}\{\{0\}} \widetilde{K}_{\xi}^{0}
\end{aligned}
$$

Proof. Let us assume that $\left(x^{0 \prime}, y_{n}^{0}, x_{n}^{0}, \xi^{0 \prime},-\xi_{n}^{0}, \xi_{n+1}^{0}\right) \notin \cup_{\tilde{\xi}_{\in} \in \Xi^{n+1} \backslash\{0\}} \tilde{K}_{\xi}^{0}$ $\times\left\{\left(\xi^{\prime},-\xi_{n}, \xi_{n+1}\right)\right\}$. Then we have the following

Lemma 4.3. There exist an open conic neighborhood $\Delta_{1}\left(\subset \Xi^{n+1}\right)$ $\{0\})$ of $\left(\xi^{0 \prime},-\xi_{n}^{0}, \xi_{n+1}^{0}\right), \tilde{\eta} \in \Gamma_{\xi^{0}}^{0}$, a neighborhood $U$ of $\left(x^{0 \prime}, y_{n}^{0}, x_{n}^{0}\right)$, positive numbers $\delta, C, t_{0}$ and a rational number a such that

$$
\begin{gathered}
x^{\prime} \cdot \eta^{\prime}-y_{n} \eta_{n}+x_{n} \eta_{n+1}<0 \quad \text { when } \quad\left(x^{\prime}, y_{n}, x_{n}\right) \in U, \\
\mid R\left(\xi^{\prime}-i\left(t|\tilde{\xi}| \eta^{\prime}+\gamma_{2} \vartheta^{\prime}\right)\right) P_{+}\left(\xi^{\prime}-i\left(t|\tilde{\xi}| \eta^{\prime}+\gamma_{2} \vartheta^{\prime}\right), \xi_{n+1}-i t|\tilde{\xi}| \eta_{n+1}\right)
\end{gathered}
$$




$$
\begin{aligned}
& \times\left. P\left(\xi^{\prime}-i\left(t|\tilde{\xi}| \eta^{\prime}+\gamma_{2} \vartheta^{\prime}\right),-\xi_{n}-i t|\tilde{\xi}| \eta_{n}\right)|\geqq \delta| \tilde{\xi}\right|^{a} \\
& \text { when } \quad \tilde{\xi} \in \Delta_{1},|\tilde{\xi}| \geqq C, 0 \leqq t \leqq t_{0},
\end{aligned}
$$

where $\gamma_{2}=\gamma_{1}+1$.

Let $\Delta$ be a conic neighborhood of $\left(\xi^{0^{\prime}},-\xi_{n}^{0}, \xi_{n+1}^{0}\right)$ such that $\bar{\Delta}$ $\{0\} \subset \Delta_{1}$. Let $\left\{\phi_{N}\left(x^{\prime}, y_{n}, x_{n}\right)\right\}$ be a bounded sequence in $C_{0}^{\infty}(U)$ which satisfies (4.1). Let $V_{t}, 0 \leqq t \leqq t_{0}$, be the chain $\tilde{\zeta}=\left(\xi^{\prime}-i\left(t \psi(\tilde{\xi})|\tilde{\xi}| \eta^{\prime}+\gamma_{2} \vartheta^{\prime}\right)\right.$, $\left.\xi_{n}+i t \psi(\tilde{\xi})|\tilde{\xi}| \eta_{n}, \xi_{n+1}-i t \psi(\tilde{\xi})|\tilde{\xi}| \eta_{n+1}\right),|\tilde{\xi}| \geqq C$, where $\psi(\tilde{\xi}) \in C^{\infty}\left(\Xi^{n+1} \backslash\{0\}\right)$ is positively homogeneous of degree zero and $\psi(\tilde{\xi})=1$ on $\Delta, \operatorname{supp} \psi \subset \Delta_{1}$, $0 \leqq \psi(\tilde{\xi}) \leqq 1$. Since supp $\phi_{N} \subset U$, by (4.7) we obtain

$$
\begin{aligned}
& \left|\mathscr{F} \phi_{N}(\tilde{\theta}-\tilde{\zeta})\right| \leqq C(C N)^{N}(1+|\tilde{\zeta}-\tilde{\theta}|)^{-N} \\
& \qquad \text { for } \tilde{\zeta} \in V_{t}, 0 \leqq t \leqq t_{0} \text { and } \tilde{\theta} \in \Xi^{n+1} .
\end{aligned}
$$

From Stokes' formula it follows that

$$
\begin{aligned}
& \mathscr{F}\left[\phi_{N} \tilde{F}\right](\tilde{\theta})=\int_{\Xi^{n+1}-i \gamma_{2} \tilde{\Xi}} \hat{\phi}_{N}(\tilde{\theta}-\tilde{\zeta}) \\
& \quad \times i^{-1} \sum_{j, h=1}^{l} \frac{R_{j k}\left(\zeta^{\prime}\right) B_{k}\left(\zeta^{\prime},-\zeta_{n}\right) \zeta_{n+1}^{j-1}}{R\left(\zeta^{\prime}\right) P_{+}\left(\zeta^{\prime}, \zeta_{n+1}\right) P\left(\zeta^{\prime},-\zeta_{n}\right)} d \tilde{\zeta} \\
& \quad=\int_{\gamma}+\int_{V_{t_{0}}} \equiv I_{1}+I_{2},
\end{aligned}
$$

where $\gamma$ is a compact chain and $\tilde{\vartheta}=(\vartheta, 0)$. In fact, Lemmas 3.2 and 4.3 imply that the integrand is holomorphic in $\cup_{0 \leqq t \leqq t_{0}} V_{t}$. Then (4.10) is obtained by (4.8), (4.9) and Stokes' formula. It is obvious that

$$
\left|I_{1}\right| \leqq C(C N)^{N}(1+|\tilde{\theta}|)^{-N} \quad \text { when } \quad \tilde{\zeta} \in \Delta .
$$

Since $|\tilde{\zeta}-\tilde{\theta}| \geqq \delta(|\tilde{\zeta}|+|\tilde{\theta}|)$ for $\tilde{\theta} \in \Delta$ and $\tilde{\zeta} \in V_{t_{0}}$, we have

$$
\left|I_{2}\right| \leqq C(C N)^{N}(1+|\tilde{\theta}|)^{-N+b} \quad \text { for } \quad \tilde{\theta} \in \Delta,
$$

where $b$ is a constant $>n+1$ and $N \geqq b$. (4.11) and (4.12) prove that $U \times \Delta \cap W F_{A}(\widetilde{F})=\varnothing$.

Q.E.D.

Proof of Lemma 4.3. (4.7) follows from the definition of $\widetilde{K}_{\xi^{\circ}}^{0}$.

(i) If $\xi^{0 \prime}=0$, then $\dot{\Sigma}_{\xi_{0}}^{0}=\dot{\Sigma}$. Thus we have 


$$
R\left(\xi^{\prime}-i\left(t|\tilde{\xi}| \eta^{\prime}+\gamma_{2} \vartheta^{\prime}\right)\right) \neq 0 \quad \text { for all } t \geqq 0
$$

(ii) When $\xi^{0 \prime} \neq 0$, it follows from Lemma 3.7 that

$$
R\left(\xi^{\prime}-i\left(t|\tilde{\xi}|^{\prime} \eta^{\prime}+\gamma_{2} \vartheta^{\prime}\right)\right) \neq 0 \quad \text { for } \quad \tilde{\xi} \in \Delta_{1},|\tilde{\xi}| \geqq C \quad \text { and } \quad 0 \leqq t \leqq t_{0} \text {. }
$$

(iii) If $\xi^{0}=0$, then $\Gamma\left(P_{\xi^{0}}, \vartheta\right)=\Gamma$. Thus we have

$$
P\left(\xi^{\prime}-i\left(t|\tilde{\xi}| \eta^{\prime}+\gamma_{2} \vartheta^{\prime}\right),-\xi_{n}-i t|\tilde{\xi}| \eta_{n}\right) \neq 0 \quad \text { for all } t \geqq 0 .
$$

(iv) When $\xi^{0} \neq 0$, it follows from Lemma 3.1 that

$$
\begin{aligned}
& P\left(\xi^{\prime}-i\left(t|\tilde{\xi}| \eta^{\prime}+\gamma_{2} \vartheta^{\prime}\right),-\xi_{n}-i t|\tilde{\xi}| \eta_{n}\right) \neq 0 \\
& \text { for } \quad \tilde{\xi} \in \Delta_{1},|\tilde{\xi}| \geqq C \text { and } 0 \leqq t \leqq t_{0}
\end{aligned}
$$

(v) If $\xi^{0^{\prime}}=0$ and $\xi_{n+1}^{0}=0$, then $\tilde{\Gamma}_{\left(\xi^{0}, \xi_{n+1}^{0}\right)}=\left\{\tilde{\zeta} \in \Xi^{n+1} ;\left(\zeta^{\prime}, \zeta_{n+1}\right) \in \Gamma(P, \vartheta)\right\}$. Thus we have

$$
P_{+}\left(\xi^{\prime}-i\left(t|\tilde{\xi}| \eta^{\prime}+\gamma_{2} \vartheta^{\prime}\right), \xi_{n+1}-i t|\tilde{\xi}| \eta_{n+1}\right) \neq 0 \quad \text { for all } t \geqq 0 .
$$

(vi) If $p_{j+}^{0}\left(\xi^{0 \prime}, \xi_{n+1}^{0}\right) \neq 0$, we have

$$
\begin{aligned}
& p_{j+}\left(\xi^{\prime}-i\left(t|\tilde{\xi}| \eta^{\prime}+\gamma_{2} \vartheta^{\prime}\right), \xi_{n+1}-i t|\tilde{\xi}| \eta_{n+1}\right) \neq 0 \\
& \text { for } \tilde{\xi} \in \Delta_{1},|\tilde{\xi}| \geqq C \text { and } 0 \leqq t \leqq t_{0}
\end{aligned}
$$

(vii) If $\xi^{0 \prime} \neq 0$ and $p_{j+}^{0}\left(\xi^{0 \prime}, \xi_{n+1}^{0}\right)=0$, then it follows from Lemma 3.1 and $\dot{\Gamma}_{\xi^{0}} \times \Xi^{2} \cap \widetilde{\Gamma}_{\left(\xi^{0}, \xi_{n+1}^{0}\right)} \subset\left\{\tilde{\zeta} \in \Xi^{n+1} ;\left(\zeta^{\prime}, \zeta_{n+1}\right) \in \Gamma\left(p_{j\left(\xi^{0}, \xi_{n+1}^{0}\right)}, \vartheta\right)\right\}$ that

$$
\begin{aligned}
& p_{j+}\left(\xi^{\prime}-i\left(t|\tilde{\xi}| \eta^{\prime}+\gamma_{2} \vartheta^{\prime}\right), \xi_{n+1}-i t|\tilde{\xi}| \eta_{n+1}\right) \neq 0 \\
& \text { for } \tilde{\xi} \in \Delta_{1},|\tilde{\xi}| \geqq C \text { and } 0 \leqq t \leqq t_{0} .
\end{aligned}
$$

(i)-(vii) and Seidenberg's lemma imply (4.8).

Q.E.D.

\section{Some Remarks and Examples}

In Theorem 2.6 the inclusion of (2.18) can not be always replaced by the equality. For the following special example we see that

(5.1) $\overline{\operatorname{ch}}\left[\cup_{j+0}^{\infty} \operatorname{supp} \widetilde{F}_{\xi^{0}, j}\left(x^{\prime}, y_{n}, x_{n}\right)\right] \subsetneq \widetilde{K}_{\tilde{\xi}^{0}} \quad$ for some $\quad \tilde{\xi}^{0} \in \Xi^{n+1} \backslash\{0\}$. 
Example 5.1 $\uparrow$ (see Example 5.1 in [9]). Put

$$
\begin{aligned}
& P(\xi)=\left(\xi_{1}^{2}-\xi_{2}^{2}-\xi_{3}^{2}\right)\left(\xi_{1}^{2}-\xi_{2}^{2} / 4-\xi_{3}^{2} / 4\right), \\
& B_{1}(\xi)=1, \quad B_{2}(\xi)=\xi_{3}^{2} .
\end{aligned}
$$

For $\tilde{\xi}^{0}=(1,1, \sqrt{3},-\sqrt{3})$ we obtain

$$
\begin{gathered}
\cup_{j=0}^{\infty} \operatorname{supp} \widetilde{F}_{\tilde{\xi}^{0}, j}=\left\{\left(x^{\prime}, y_{3}, x_{3}\right) \in \mathbb{R}^{2} \times \mathbb{R}_{+}^{1} \times \mathbb{R}_{+}^{1} ; x_{1}=4\left(x_{3}+y_{3}\right) / \sqrt{3},\right. \\
\left.x_{2}=-\left(x_{3}+y_{3}\right) / \sqrt{3}\right\} .
\end{gathered}
$$

$$
\begin{array}{r}
\widetilde{K}_{\tilde{\zeta}^{0}}=\left\{\left(x^{\prime}, y_{3}, x_{3}\right) \in \mathbb{R}^{2} \times \mathbb{R}_{+}^{1} \times \mathbb{R}_{+}^{1} ; x_{1}=4\left(x_{3}+y_{3}\right) / \sqrt{3}+u,\right. \\
\left.x_{2}=-\left(x_{1}+y_{3}\right) / \sqrt{3}-u, u \geqq 0\right\} .
\end{array}
$$

In this example the lateral waves do not appear.

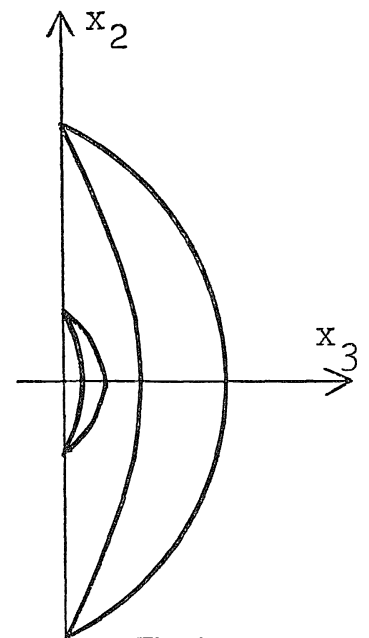

Fig. 1.

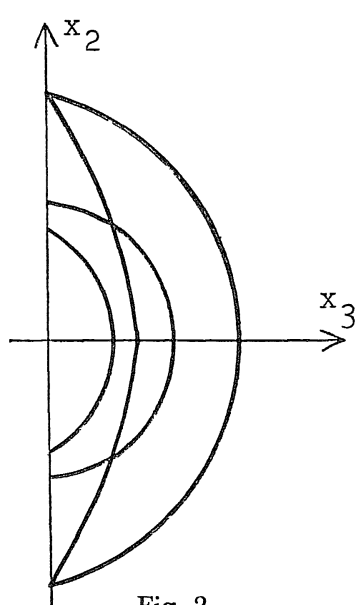

Fig. 2.

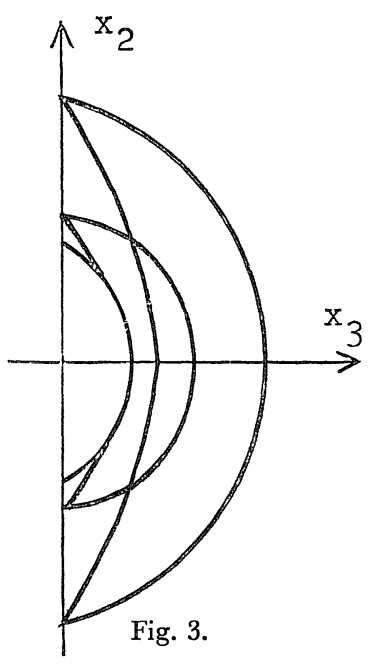

Fig. 1 illustrates the intersection of $\cup_{\tilde{\xi} \in \Xi^{4} \mid\{0\}} \cup_{j=0}^{\infty} \operatorname{supp} \tilde{F}_{\tilde{\xi}, j}\left(x^{\prime}, y_{3}, x_{3}\right)$ with the plane $x_{1}=c$, where $y_{3}=1$ and $2<c \leqq 4 \sqrt{3} / 3$. This also illustrates that of $\cup_{\tilde{\xi} \in \Xi^{4} \mid\{0\}} \tilde{K}_{\tilde{\xi}}$ with the plane $x_{1}=c$, where $y_{3}=1$ and $2<c \leqq$ $4 \sqrt{3} / 3$. Fig. 2 illustrates that of $\cup_{\tilde{\xi} \in \Xi^{4} \backslash\{0\}} \cup_{j=0}^{\infty} \operatorname{supp} \tilde{F}_{\tilde{\xi}, j}\left(x^{\prime}, y_{3}, x_{3}\right)$ with the plane $x_{1}=c$, where $y_{3}=1$ and $c>4 \sqrt{3} / 3$. Fig. 3 illustrates that of $\cup_{\tilde{\xi} \in \Xi^{4} \mid\{0\}} \tilde{K}_{\tilde{\xi}}$ with the plane $x_{1}=c$, where $y_{3}=1$ and $c>4 \sqrt{3} / 3$.

$\dagger$ This example was given by T. Shirota. 
In Theorem 4.1 of [9] it was proved that $\dot{\Sigma}_{\xi 0}^{0}=\dot{\Sigma}_{\xi 0}$, if the following conditions are satisfied: (i) Each $p_{j}^{0}\left(\xi^{0 \prime}, \mu\right)=0$ has no real multiple roots. (ii) $\left\{P(-D), B_{j}(-D)\right\}$ satisfies the Lopatinski condition. However, for the following example we observe that $\dot{\Sigma}_{\xi 0}^{0}, \subsetneq \dot{\Sigma}_{\xi^{0}}$, and $\widetilde{K}_{\tilde{\xi}}^{0} \supsetneq \widetilde{K}_{\tilde{\xi} 0}$ for some $\tilde{\xi}^{0} \in \Xi^{n+1} \backslash\{0\}$.

Example 5.2. Put

$$
\begin{gathered}
P(\xi)=\left(\xi_{1}^{2}-\xi_{2}^{2}-\xi_{3}^{2}+a \xi_{2}+b\right)\left(\left(\xi_{1}-\xi_{2}\right)^{2}-\xi_{3}^{2}\right), \quad a>0, b \in \mathbb{C}, \\
B_{1}(\xi)=1, \quad B_{2}=\left(-\xi_{1}+(1-i) \xi_{2}\right) \xi_{3}-\xi_{3}^{2} .
\end{gathered}
$$

Then

$$
R\left(\xi^{\prime}\right)=i \check{\zeta}_{2}+\sqrt[+]{\xi_{1}^{2}-\xi_{2}^{2}+a \xi_{2}+b}
$$

where $\sqrt[+]{\xi_{1}^{2}-\xi_{2}^{2}+a \xi_{2}+b}$ denotes the branch of $\sqrt{-}$ with positive imaginary part when $-\operatorname{Im} \xi_{1}$ is large enough and $\xi_{2}$ is real. It is easy to see that $\left\{P(-D), B_{j}(-D)\right\}$ does not satisfy the Lopatinski condition. For $\xi^{0 \prime}=(0,-1)$ we have

$$
Q_{0}^{0}\left(\eta^{\prime}\right)=i a / 2, \quad \widetilde{R}_{0 \xi^{0}}\left(\eta^{\prime}\right)=-i \eta_{1}^{2} / 2 .
$$

Thus

$$
\dot{\Sigma}_{\xi^{0}}^{0}=\left\{\eta^{\prime} \in \Xi^{2} ; \eta_{1}>0\right\}, \quad \dot{\Sigma}_{\xi^{0}}=\Xi^{2} .
$$

For $\xi^{0}=(0,-1,1,-1)$ we have also

$$
\widetilde{K}_{\xi}^{0} \supseteqq \widetilde{K}_{\tilde{\xi}} .
$$




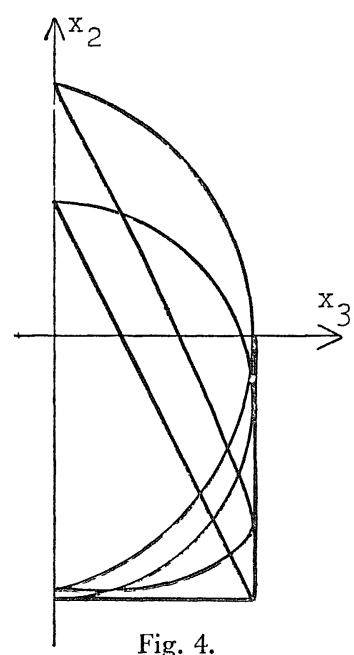

Fig. 4.

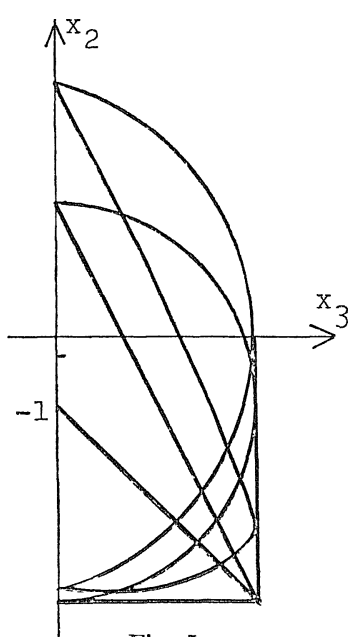

Fig. 5.

Fig. 4 illustrates the intersection of $\cup_{\tilde{\xi} \in \Xi^{4} \mid\{0\}} \widetilde{K}_{\xi}$ with the planc $x_{1}=$ const. $>0$, where $y_{3}=1$. Fig. 5 illustrates that of $\cup_{\tilde{\xi} \in E^{4} \mid\{0\}} \widetilde{K}_{\tilde{\xi}}^{0}$ with the plane $x_{1}=$ const. $>0$, where $y_{3}=1$.

The above example shows that the localization of the principal part of the Lopatinski determinant does not always coincide with the principal part of the localization of the Lopatinski determinant.

\section{References}

[1] Andersson, K. G., Propagation of analylicity of solutions of partial differcntial equations with constant coefficients, Ark. Mat., 8 (1971), 277-302.

[2] Atiyah, M. F., Bott, R. and Gårding, L., Lacunas for hyperbolic differential operators with constant coefficients I, Acta Math., 124 (1970), 109-189.

[3] Duff, G. F. D., On wave fronts, and boundary waves, Comm. Pure Appl. Math., 17 (1964), 189-225.

[4] Hörmander, L., On the singularities of solutions of partial differential equations, International Conference of Functional Analysis and Related Topics. Tokyo 1969.

[5] —- Uniqueness theorems and wave front sets for solutions of linear differential equations with analytic coefficients, Comm. Pure Appl. Math., 24 (1971), 671-703.

[6] Matsumura, M., Localization theorem in hyperbolic mixed problems, Proc. Japan Acad., 47 (1971), 115-119.

[7] Sakamoto, R., $\mathscr{E}$-well posedness for hyperbolic mixed problems with constant coefficients, J. Math. Kyoto Univ., 14 (1974), 93-118.

[8] Tsuji, M., Fundamental solutions of hyperbolic mixed problems with constant coefficients, Proc. Japan Acad., 51 (1975), 369-373.

[9] Wakabayashi, S., Singularities of the Riemann functions of hyperbolic mixed problems in a quarter-space, Publ. RIMS, Kyoto Univ., $\mathbb{1 1}$ (1976), 417-440. 
